OPEN ACCESS

Edited by:

Partha Basu,

Duquesne University, USA

Reviewed by:

Mustafa Yucel,

Middle East Technical University,

Turkey

Eric D. Van Hullebusch, University Paris-Est, France

*Correspondence: Patrick Billard patrick.billard@univ-lorraine.fr

Specialty section: This article was submitted to Microbiological Chemistry and

Geomicrobiology,

a section of the journal

Frontiers in Microbiology

Received: 23 December 2015 Accepted: 16 March 2016

Published: 31 March 2016

Citation:

Parrello D, Zegeye A, Mustin C and Billard P (2016) Siderophore-Mediated

Iron Dissolution from Nontronites is

Controlled by Mineral Cristallochemistry.

Front. Microbiol. 7:423.

doi: 10.3389/fmicb.2016.00423

\section{Siderophore-Mediated Iron Dissolution from Nontronites Is Controlled by Mineral Cristallochemistry}

\author{
Damien Parrello ${ }^{1,2}$, Asfaw Zegeye ${ }^{1}$, Christian Mustin ${ }^{1}$ and Patrick Billard ${ }^{1 *}$ \\ ${ }^{1}$ Laboratoire Interdisciplinaire des Environnements Continentaux, UMR 7360 Centre National de la Recherche Scientifique - \\ Université de Lorraine, Vandœuvre-lès-Nancy, France, ${ }^{2}$ Civil and Environmental Engineering, University of Missouri, \\ Columbia, MO, USA
}

Bacteria living in oxic environments experience iron deficiency due to limited solubility and slow dissolution kinetics of iron-bearing minerals. To cope with iron deprivation, aerobic bacteria have evolved various strategies, including release of siderophores or other organic acids that scavenge external Fe(III) and deliver it to the cells. This research investigated the role of siderophores produced by Pseudomonas aeruginosa in the acquisition of $\mathrm{Fe}$ (III) from two iron-bearing colloidal nontronites (NAu-1 and NAu-2), comparing differences in bioavailability related with site occupancy and distribution of $\mathrm{Fe}$ (III) in the two lattices. To avoid both the direct contact of the mineral colloids with the bacterial cells and the uncontrolled particle aggregation, nontronite suspensions were homogenously dispersed in a porous silica gel before the dissolution experiments. A multiparametric approach coupling UV-vis spectroscopy and spectral decomposition algorithm was implemented to monitor simultaneously the solubilisation of Fe and the production of pyoverdine in microplate-based batch experiments. Both nontronites released $\mathrm{Fe}$ in a particle concentration-dependent manner when incubated with the wild-type $P$. aeruginosa strain, however iron released from NAu-2 was substantially greater than from NAu-1. The profile of organic acids produced in both cases was similar and may not account for the difference in the iron dissolution efficiency. In contrast, a pyoverdine-deficient mutant was unable to mobilize Fe(III) from either nontronite, whereas iron dissolution occurred in abiotic experiments conducted with purified pyoverdine. Overall, our data provide evidence that $P$. aeruginosa indirectly mobilize Fe from nontronites primarily through the production of pyoverdine. The structural Fe present on the edges of NAu-2 rather than NAu-1 particles appears to be more bio-accessible, indicating that the distribution of $\mathrm{Fe}$, in the tetrahedron and/or in the octahedron sites, governs the solubilisation process. Furthermore, we also revealed that $P$. aeruginosa could acquire iron when in direct contact with mineral particles in a siderophore-independent manner.

Keywords: siderophore, pyoverdine, Pseudomonas aeruginosa, bioweathering, iron mobilization, nontronite, clay 


\section{INTRODUCTION}

Iron is an essential nutrient, playing a central role in the growth and the development of most living organisms. It is required for several fundamental enzymatic processes including respiration, photosynthesis and DNA synthesis. Most microorganisms require $\mathrm{Fe}$ concentration around $10^{-7}$ to $10^{-5} \mathrm{M}$ to support optimal growth (Andrews et al., 2003). Although widespread in the environment, $\mathrm{Fe}$ is very insoluble in oxic conditions at circumneutral $\mathrm{pH}$, which tends to limit the concentration of dissolved Fe (Cornell and Schwertmann, 2004). For instance the solubility products of common iron oxides such as goethite, hematite and ferrihydrite are extremely low $\left(\left(\mathrm{Fe}^{3+}\right) \cdot(\mathrm{OH})^{3}=\right.$ $10^{-39}-10^{-44} \mathrm{M}$ ), thus the bioavailable Fe concentration falls much below the metabolic needs of bacteria. To overcome the low abundance of free $\mathrm{Fe}$ in aerobic environments, bacteria have developed different strategies such as the excretion of protons and/or low molecular weight organic acids (e.g., citrate, oxalate) (Banwart et al., 1989). These metabolites contribute to mineral weathering by decreasing the $\mathrm{pH}$ or by forming complexes with surface ions and subsequently solubilizing the Fe from the mineral lattice by either proton-promoted or ligand-promoted dissolution (Balland et al., 2010).

The synthesis and the excretion of siderophores is another important avenue used by bacteria for $\mathrm{Fe}$ acquisition in ironlimited environments (Powell et al., 1980; Saha et al., 2013). Siderophores are low molecular weight organic molecules, with extremely high affinities for $\mathrm{Fe}$ (III) up to $10^{52} \mathrm{M}^{-1}$ and as such they are capable to solubilize $\mathrm{Fe}(\mathrm{III})$ from minerals lattices. These multi-dentate ligands are considered as one of the main molecules involved in the $\mathrm{Fe}$ uptake in the rhizosphere (Powell et al., 1980; Hider and Kong, 2010; Schalk and Guillon, 2013). For instance, Pseudomonas aeruginosa, a Gram-negative bacterium commonly found in soil and water, excretes two major siderophores, pyoverdine and pyochelin, under iron deficient conditions (Cox et al., 1981; Brandel et al., 2012; Schalk and Guillon, 2013). Pyoverdine is a dihydroxyquinoline-derived chromophore to which a short peptide chain is attached which binds $\mathrm{Fe}^{3+}$ in a 1:1 stoichiometry with a stability constant for $\mathrm{Fe}(\mathrm{III})$ around $10^{32}$ $\mathrm{M}^{-1}$ (Albrecht-Gary et al., 1994). On the other hand pyochelin is derivate from thiazoline [2(2-o-hydroxy-phenyl-2-thiazolin-4yl)-3-methylthiazolidine-4-carboxylic acid)] (Cox et al., 1981), which binds $\mathrm{Fe}^{3+}$ in a 2:1 stoichiometry with a stability constant for $\mathrm{Fe}(\mathrm{III})$ around $10^{17} \mathrm{M}^{-1}$ (Brandel et al., 2012).

The dissolution of $\mathrm{Fe}(\mathrm{III})$ bearing minerals such as iron oxides, clay minerals, triggered by siderophores has been the subject of numerous studies (Albrecht-Gary and Crumbliss, 1998; Cheah et al., 2003; Kraemer, 2004; Dehner et al., 2011) and have highlighted different key parameters. For instance, efficiency of $\mathrm{Fe}(\mathrm{III})$ uptake by siderophores was enhanced in the presence of organic acids such as oxalate, a common metabolite resulting from bacterial activity. This suggested synergic action consisted, on the one hand, in the labialization of the structural Fe by oxalate and on the other hand, in the detachment of the labile pool of $\mathrm{Fe}(\mathrm{III})$-oxalate surface complexes by siderophores, thus driving the reaction toward more dissolution (Kraemer, 2004).
Interestingly, an additive effect rather than a synergic one was observed when ascorbate, a reducing agent, was used instead of oxalate, showing the complex interplay between siderophores and organic molecules during the dissolution of structural $\mathrm{Fe}(\mathrm{III})$ (Cheah et al., 2003; Dehner et al., 2010). Additionally, Dehner et al. (2011) have shown that the uptake of Fe(III) by $P$. mendocina from hematite was governed by the particles size. Indeed, $\mathrm{Fe}(\mathrm{III})$ associated with particles less than a few tens of $\mathrm{nm}$ was more bioavailable than $\mathrm{Fe}$ (III) associated with larger particles emphasizing the significance to probe particle size when assessing bioavailability. Together these studies stressed that mineral weathering was a combination of various and complex set of interactions between the mineral and the bacteria. These complex biotic and abiotic interactions likely overcome thermodynamic and kinetic constraints imposed by the mineralogy, the texture and crystallo-chemistry of the mineral, leading to the release of structural Fe, which becomes available for microbial uptake.

Although iron oxides are the ultimate source of $\mathrm{Fe}$, iron bearing clay minerals can also play the role of $\mathrm{Fe}$ pool for bacterial metabolism. Recent experiments showed that structural Fe(III) in smectite, a 2:1 phylosilicate, was available for bacterial uptake (Haack et al., 2008; Kuhn et al., 2013; Ferret et al., 2014). However, there still remains a gap in our knowledge whether the structural $\mathrm{Fe}$ (III) site occupancy influences the bioavailability of Fe. NAu-1 and NAu-2 are two nontronite, hydrous $\mathrm{Fe}(\mathrm{III})$ bearing dioctahedral clay minerals. They are 2:1 phyllosilicates of similar composition with two tetrahedral sheets per octahedral sheet. The structural $\mathrm{Fe}(\mathrm{III})$ are located in both the tetrahedrons and in the octahedrons for the two nontronites, the main difference being that $2 \%$ of total iron is located in the tetrahedral sheet for NAu- 1 against $8 \%$ for NAu-2.

The aim of this study was therefore to study the bioaccessibility of structural $\mathrm{Fe}$ in these two nontronites, NAu-1 and NAu-2, to determine whether crystallographic sites (tetrahedrons vs. octahedrons) control its uptake by Pseudomonas aeruginosa PAO1.

\section{MATERIALS AND METHODS}

\section{Bacterial Strains, Plasmids, and Culture Conditions}

The following $P$. aeruginosa PAO1 strains were used in this study: PAO1 wild-type (ATCC 15692), PAO1- $\Delta p v d A$ (Ochsner et al., 2002) deficient in pyoverdine production and PAO1 $\Delta p v d D$ $\triangle p c h E F$ (Ghysels et al., 2004) impaired in the production of both pyoverdine and pyochelin. E. coli DH10B served as a host for cloning purposes. The plasmid pPROBE-bfrB which contains an iron responsive $b f r B-g f p$ gene fusion was constructed as described previously (Parrello et al., 2015) except that the bfrB promoter region was cloned in pPROBE-NT (Miller et al., 2000). Bacteria were routinely grown aerobically on LB agar or in LB broth at $37^{\circ} \mathrm{C}$. Deferrated Casamino Acids medium (DCAA medium) (Visca et al., 1992) was used for mineral weathering experiments. When required, the medium was supplemented with kanamycin at final concentrations of $40 \mu \mathrm{g} \cdot \mathrm{mL}^{-1}$ for $E$. coli or $500 \mu \mathrm{g} \cdot \mathrm{mL}^{-1}$ for $P$. aeruginosa. 


\section{Preparation of Nontronite Embedded in Hybrid Silica Gels}

Two nontronites, NAu-1 and NAu-2, purchased from the Source Clays Minerals repository at Purdue University were used for bioweathering experiments. NAu-1 and NAu-2 contain Fe(III) in different structural sites and are defined by the structural formula $\left(\mathrm{Si}_{6.98} \mathrm{Al}_{0.95} \mathrm{Fe}_{0.07}\right)\left(\mathrm{Al}_{0.36} \mathrm{Fe}_{3.61} \mathrm{Mg}_{0.04}\right) \mathrm{O}_{20}(\mathrm{OH})_{4} \mathrm{Na}_{1.05}$ and $\left(\mathrm{Si}_{7.55} \mathrm{Al}_{0.16} \mathrm{Fe}_{0.29}\right) \quad\left(\mathrm{Al}_{0.34} \mathrm{Fe}_{3,54} \mathrm{Mg}_{0.05}\right) \mathrm{O}_{20}(\mathrm{OH})_{4} \mathrm{Na}_{0.72}$, respectively. The average size of the particles used in the present study was $460 \mathrm{~nm}$ in length, $60 \mathrm{~nm}$ in width for NAu-1, 368 $\mathrm{nm}$ in length, $93 \mathrm{~nm}$ in width for NAu-2, and around $1 \mathrm{~nm}$ thickness for both nontronites (Michot et al., 2008). Hybrid silica gels were prepared by immobilization of nontronite particles in a microporous, tetraethylorthosilicate- (TEOS, Fluka) derived silica gels by a sol-gel technique as described previously (Grybos et al., 2010, 2011; Oulkadi et al., 2014). For this study, the procedure was automated and silica gels were produced in 96wells microplates (MTP 96/F-bottom, Eppendorf) with the use of epMotion 5070's automated pipetting system (Eppendorf). The gels had a volume of $0.16 \mathrm{ml}$ and contained $3.3 \mathrm{~g} \mathrm{~L}^{-1}$ of either $\mathrm{NAu}-1$ or NAu-2, or both nontronites at concentrations ranging from 0 to $3.3 \mathrm{~g} \mathrm{~L}^{-1}$ and $\mathrm{NAu}-1 / \mathrm{NAu}-2$ ratios ranging from 0 to $100 \%$.

\section{Bioweathering Experiments}

The nontronite weathering ability of $P$. aeruginosa was determined by incubating bacteria with nontronite embedded in hybrid silica gels in different conditions and by further measurement of iron release and production of organic acids and pyoverdine. $P$. aeruginosa strains were grown overnight in LB broth. The cells were collected by centrifugation, washed twice with DCAA and the cell pellets were suspended in the same medium at an optical density at $600 \mathrm{~nm}\left(\mathrm{OD}_{600}\right)$ of 1 .

To measure the kinetics of organic acids production, suspensions of PAO1 WT and $\triangle p v d A$ mutant strains were separately inoculated to an $\mathrm{OD}_{600}$ of 0.1 in $4 \mathrm{ml}$ of DCAA medium supplemented with four $0.16 \mathrm{ml}$ silica gel plugs containing NAu-1 or NAu-2 particles at $3.3 \mathrm{~g} \mathrm{~L}^{-1}$ Incubations were performed in triplicate at $37^{\circ} \mathrm{C}$ with shaking at $260 \mathrm{rpm}$ for $60 \mathrm{~h}$. After 2, 6, 12, 36, and $60 \mathrm{~h}$ of incubation, $100 \mu \mathrm{l}$ of each culture were diluted in $900 \mu \mathrm{l}$ of ultrapure water and centrifuged at $13,500 \times \mathrm{g}$ for $5 \mathrm{~min}$. Supernatants were then filtered with $0.22 \mu \mathrm{m}$ pore size filters (Millipore) and stored at $-20^{\circ} \mathrm{C}$ before organic acids quantification.

To assess the respective role of nontronite structure and pyoverdine production in the bio-weathering process, suspensions of PAO1 WT and $\triangle p v d A$ mutant strains were inoculated to $\mathrm{OD}_{600}$ of 0.1 in tubes with $2 \mathrm{ml}$ of DCAA and four silica gels plugs containing 25 combined concentrations (i.e., $0,0.83,1.65,2.48$, and $3.3 \mathrm{~g} \mathrm{~L}^{-1}$ ) and ratios (i.e., $0,25,50,75$, and $100 \%$ ) of NAu- $1 / \mathrm{NAu}-2$ particles. The suspensions were incubated at $37^{\circ} \mathrm{C}$ with shaking for $24 \mathrm{~h}$ before determination of iron and pyoverdine concentration. This experiment was conducted in duplicate.

\section{Analytical Methods}

Organic acids were quantified from weathering solutions using an ion chromatograph (ICS 3000, Dionex corp.) equipped with an Ion $\mathrm{Pac}^{\circledR}$ column (AS $11 \mathrm{HC}$, Dionex corp.) according to a $\mathrm{KOH}$ gradient of $0.9-60 \mathrm{mM}$ over $50 \mathrm{~min}$ at a flow rate of $1.3 \mathrm{~mL}$ per min. Standard solutions $(0.1 \mathrm{mM})$ used were sodium salts of acetate, citrate, formate, gluconate, D, Lmalate, oxalate, propionate, succinate, (Sigma Aldrich), lactate, and malonate (Fluka). Measurement uncertainty was lower than $0.5 \%$ for all organic acids and the detection limit was close to $0.1 \mathrm{ppm}$.

Iron concentration in weathering solutions was measured spectrophotometrically at $565 \mathrm{~nm}$ by adding $20 \mu \mathrm{l}$ of Ferrospectral (Merck, Millipore) dye indicator to $200 \mu l$ of $0.22 \mu \mathrm{m}$ filtered culture supernatants. The fraction of iron adsorbed onto silica gels and embedded on mineral surfaces was estimated by treating 1 volume of silica gel with 2 volumes of $\mathrm{KCl}(1 \mathrm{M})$. After $1 \mathrm{~h}$ of incubation at $28^{\circ} \mathrm{C}$, Ferrospectral dye indicator was added as described above. The fraction of iron precipitated onto silica and mineral surfaces was estimated by treating 1 volume of silica gel with 2 volumes of hydroxylamine solution $\left(0.2 \% \mathrm{NH}_{2} \mathrm{OH}\right.$ in $\left.\mathrm{KCl} 1 \mathrm{M}\right)$ for $1 \mathrm{~h}$ at $28^{\circ} \mathrm{C}$ before addition of Ferrospectral.

Concentration of pyoverdine in culture supernatants was quantified spectrophotometrically by measuring the absorbance at $400 \mathrm{~nm}$ with $\varepsilon=19000 \mathrm{M}^{-1} \mathrm{~cm}^{-1}$ (Meyer and Abdallah, 1978).

In addition to the above described mono-wavelength measurement of iron and pyoverdine, the production patterns for these two compounds were determined by means of a recently developed approach that couples spectroscopy with blind spectral decomposition of the spectral data by the Candecomp/Parafac (CP) model (Parrello et al., 2015). Briefly, $200 \mu \mathrm{l}$ of filtered supernatants from cultures grown with different concentrations and ratios of NAu-1/NAu-2 particles were transferred in 96 well microplates. For pyoverdine profiling, changes in absorption spectra were monitored by scanning the samples from 300 to $900 \mathrm{~nm}$ in $2 \mathrm{~nm}$ steps and with a $2 \mathrm{~nm}$ bandwidth using a FLX-Xenius spectrophotometer (SAFAS, Monaco). For iron dissolution profiling, similar absorbance spectra were performed on both culture supernatants and solutions from $\mathrm{KCl}$ and hydroxylamine-treated silica gel plugs after Ferrospectral addition. Raw absorption data were collected and exported for further signal processing with Candecomp/Parafac algorithms running under Matlab software.

\section{Blind Spectral Decomposition by Candecomp/Parafac}

The multi-way analysis of spectral data by $\mathrm{CP}$ algorithm provided multilinear decomposition of a data matrix without any a priori information about the spectra of the absorbing compounds. It was assumed that absorption spectra from different batches are indicative of various mixtures of $\mathrm{R}$ components. Each of the $\mathrm{R}$ absorbing component spectrum, termed as the rth source $(\mathrm{r}=$ $1, \ldots, R)$, is mathematically represented by a vector $s_{\mathrm{r}}=\left[\mathrm{s}_{\mathrm{r} 1} \ldots\right.$ $\left.\mathrm{s}_{\mathrm{rN}}\right]^{\mathrm{T}}$ made of $\mathrm{N}$ entries, i.e., the absorption intensities at each 
wavelength. The acquisition of absorption spectra as a function of two crossed parameters (i.e., the concentration of nontronite and the NAu-1/NAu-2 ratio in silica gels) generated a threeway data array $\mathcal{X}$ (three-order tensor; nontronite concentration $\mathrm{x} \mathrm{NAu}-1 / \mathrm{NAu}-2$ ratio $\mathrm{x}$ wavelength), which can be expressed by the following tri-linear $\mathrm{CP}$ mode:

$$
\mathcal{X}_{m, p, n}=\sum_{r=1}^{R} a_{r m} b_{r p} s_{r n}+\mathcal{E}_{m, p, n}
$$

where $R$ is the number of spectral sources (i.e., decomposition rank) and $\mathcal{E}$ is the residual error term (three-order tensor) including experimental error, signal noise or "non-linear" component behavior. An equivalent representation of Equation (1) is given by:

$$
\mathcal{X}=[[\mathbf{A}, \mathbf{B}, \mathbf{S}]]+\mathcal{E}
$$

where the three matrices $\mathbf{A}(M$-by- $R), \mathbf{B}(P$-by- $R)$ and $\mathbf{S}(N$-by$R)$ are respectively obtained by stacking the vectors $\mathbf{a}_{r}, \mathbf{b}_{r}$ and $\mathbf{s}_{r}$. The matrices $\mathbf{A}$ and $\mathbf{B}$ in Equation (2) characterize the behavior of the R sources according to the two experimental parameters. The matrix $\mathbf{S}$ characterizes the spectral shape of the identified sources. The rank $R$ of decomposition is assessed experimentally by observing the decrease of the energy of the energy component $\mathcal{E}$. The retained value is obtained after successive trials using possible values of $R$ around the value of $R$ found before. The energy component represents the level of contribution of each source in the explained variation (the fitting accuracy of the raw three-way dataset). Thus we can soundly define that if the nth supplementary source is 2 log lower the most explicative source, the optimal number of sources is $n-1$.

The main interest of the CP decomposition comes from its good uniqueness properties, meaning that the matrices $\mathbf{A}, \mathbf{B}$, and $\mathbf{S}$ can be uniquely estimated from the data $\mathcal{X}$, i.e., the proposed mathematical model actually describes what happens physically. Another very interesting feature of the $\mathrm{CP}$ decomposition is the possibility to extract signals of interest from the background noise, allowing the analysis of spectra obtained from complex environments.

\section{Microscopy and Image Analysis}

The fluorescence of individual cells of PAO1 $\triangle p v d D \triangle p c h E F /$ pPROBE-bfrB cultured for $48 \mathrm{~h}$ in DCAA medium containing colloidal suspensions of either NAu-1 and NAu-2 $\left(3.3 \mathrm{~g} \mathrm{~L}^{-1}\right)$ was quantified by fluorescence microscopy. Images were acquired on a Nikon Eclipse 80i microscope with Plan Fluor X 40 objective and FITC (ex: $465-495 \mathrm{~nm}$; em: 515-555 nm) filter, and equipped with an Intensilight C-HGFiE fluorescence light source and a digital sight DS-Qi1MC camera. Quantification of GFP fluorescence was performed using the NIS-Elements BR software (version 3.2, Nikon) by encircling bacterial cells and measuring the mean pixel intensity. Image adjustments such as changes of contrast and brightness were applied equally across the entire image.

\section{RESULTS AND DISCUSSION \\ Bio-dissolution of Structural Iron of Colloidal Size NAu-1 and NAu-2}

Iron bio-dissolution from colloidal NAu-1 and NAu-2 entrapped in a porous silica gel was investigated using a multi-parametric approach where the NAu-1 and NAu-2 ratio was varied across a range of particle concentrations. Previous experiments indicated that the mineral trapping procedure neither affected the mineral structure nor prompted its dissolution as no iron was detected in the media in the absence of bacteria (Grybos et al., 2010, 2011). Additionally, the nontronite particles included in the silica gel were homogeneously dispersed and in a colloidal form, thus the reactivity of the mineral was not likely affected by a change of its surface area (due to aggregation), or to physical defects (stacking faults, mixed layering). Another critical feature was the absence of physical contact between the mineral and the bacteria cells once the mineral was trapped in the silica gel (Grybos et al., 2010, 2011). Therefore, this experimental approach allowed assessing the dissolution of $\mathrm{Fe}(\mathrm{III})$ from colloidal NAu-1 and NAu-2 without a direct contact with $P$. aeruginosa cells. The dissolution, if any, would be more likely related to the diffusion of organic molecules through the micro-porous silica gel.

The multi-way approach consisted of 25 independent assays where the nontronite particle concentrations and NAu-1/NAu-2 ratio were varied all together and incubated with $P$. aeruginosa for $24 \mathrm{~h}$ before spectrophotometric measurement of iron release. For instance, Figure 1A represents the Fe dissolution profile when the assays were run with a constant nontronite ratio (100\% of NAu-2 with [NAu-1]/[NAu-1] + [NAu-2] $=0 \%)$ across a range of particle concentrations ( 0 to $\left.3.3 \mathrm{~g} \mathrm{~L}^{-1}\right)$ in the presence of PAO1 WT. Figure 2A, on the other hand, illustrates the Fe dissolution profile when the assays were run with a constant particle concentration (i.e., $3.3 \mathrm{~g} \mathrm{~L}^{-1}$ ) while varying NAu-1 and NAu-2 ratio (i.e., $0 \leq[\mathrm{NAu}-1] /[\mathrm{NAu}-1]+[\mathrm{NAu}-2] \leq 100 \%$ ) in the presence of the same strain. An increase of Fe released in the culture medium was observed with increasing particle concentration indicating that the diffusion of the compounds responsible for the dissolution of $\mathrm{Fe}$, through the porous silica gel was not a limiting factor in the range of tested particle concentrations (Figure 1A). For the assay run with the highest particle concentration (i.e., $3.3 \mathrm{~g} \mathrm{~L}^{-1}$ ) a total of $5.18 \pm 0.21 \mu \mathrm{M}$ of Fe were released in the culture media, $58 \%$ of which were soluble Fe. The same trend (soluble over total $\mathrm{Fe}$ ) was noted across the entire particles concentrations tested (Figure 1A). The presence of Fe extracted with $\mathrm{KCl}$ and hydroxylamine treatment indicated that a small fraction of iron was adsorbed and/or precipitated on the mineral and/or on the silica gel after dissolution (Figure 1A). Overall the results show that a substantial amount of Fe was liberated in the culture media. Given the fact that NAu particles are highly insoluble at circumneutral $\mathrm{pH}$, the $\mathrm{Fe}$ present in the media was likely chelated by organic molecules (i.e., excreted by bacteria), thus becoming available for bacterial uptake. Similarly, the related strain Pseudomonas mendocina ymp requires micromolar concentration of $\mathrm{Fe}$ for optimal growth (Dehner et al., 2010). Likewise, the amount of Fe dissolved from 

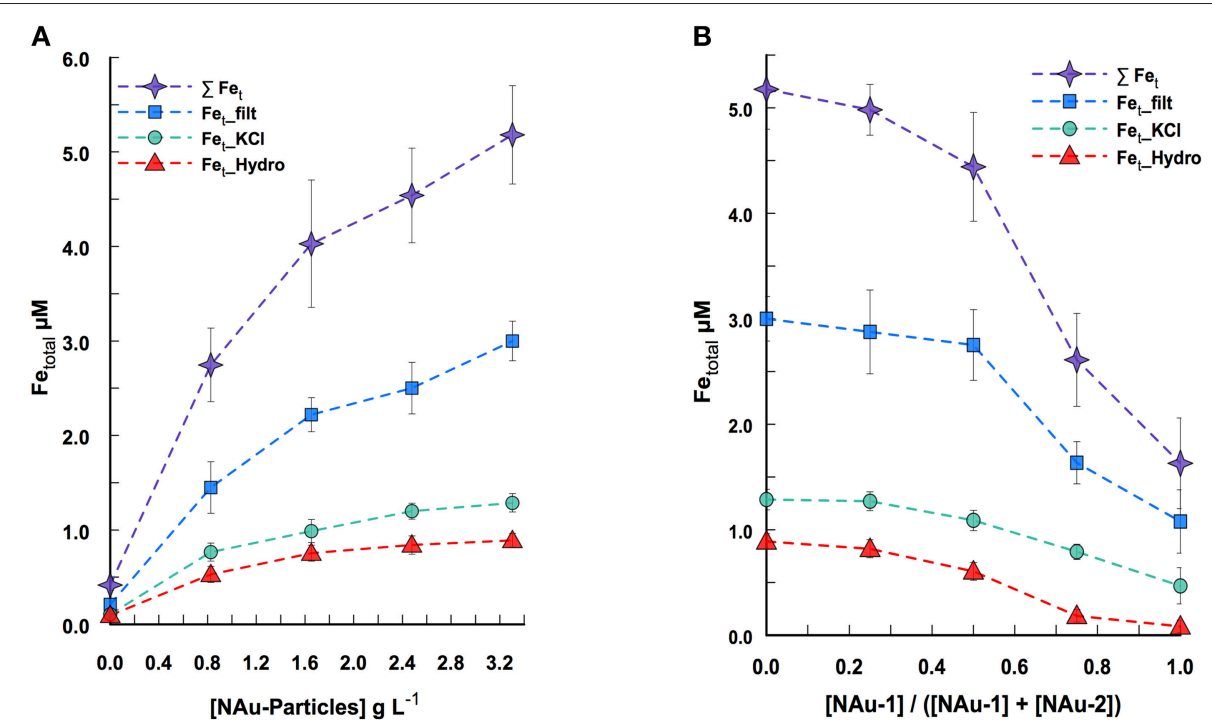

FIGURE 1 | $P$. aeruginosa-promoted Fe dissolution from silica gel-embedded nontronites in a system where NAu-1 to NAu-2 ratios are varied across a range of particle concentration, after $\mathbf{2 4} \mathbf{~ h}$ of incubation. (A) Fe dissolution for a constant NAu-1 to NAu-2 ratio ([NAu-1]/([NAu-1] + [NAu-2]) $=0$ ) while the particle concentration varied. (B) Fe dissolution for a constant particle concentration $\left(3.3 \mathrm{~g} \mathrm{~L}^{-1}\right)$ with different NAu-1 to NAu-2 ratios. The symbols represent soluble $\mathrm{Fe}$ (squares), $\mathrm{KCl}$ extracted Fe (circles), hydroxylamine- $\mathrm{KCl}$ extracted Fe (triangles) and the sum of the three above Fe concentrations (stars). Error bars indicate the standard error of the mean ( $n=2$ for soluble Fe; $n=3$ for $\mathrm{KCl}$ - and hydroxylamine- $\mathrm{KCl}$ extracted Fe).

NAu-2 in the culture media is sufficient to support $P$. aeruginosa growth (not shown).

A significant amount of the released $\mathrm{Fe}(\approx 60 \%)$ was present in solution for the different ratios tested, indicating that the variation of NAu-1 and NAu-2 ratio did not increase or affect the adsorbed and/or the precipitated Fe fraction (Figure 1B). A two-trend pattern was observed during the release of Fe while varying the ratio: (i) in the ratio range of $0 \% \leq[\mathrm{NAu}-1] /[\mathrm{NAu}-1]$ $+[\mathrm{NAu}-2] \leq 50 \%$, the total amount of dissolved Fe decreased from $5.18 \pm 0.21 \mu \mathrm{M}$ to $4.44 \pm 0.33 \mu \mathrm{M}$ (i.e., a decrease of $\approx 15 \%$ ) and (ii) in the range of $50 \% \leq$ [NAu-1]/[NAu-1] + [NAu-2] $\leq 100 \%$ a higher decreased was registered from 4.44 $\pm 0.33 \mu \mathrm{M}$ to $1.63 \mu \mathrm{M} \pm 0.30$ (i.e., a decrease of $\approx 65 \%$ ). The data indicate that $P$. aeruginosa did not mobilize as much Fe in the presence of NAu- 1 than NAu-2. Because NAu-1 and NAu-2 were in a colloidal form in the silica gel and had almost the same surface area, their respective reactivity would not be affected by a change of surface area due to aggregation. Thus, the difference of reactivity observed between the two forms of nontronite is based on their crystallography distinctiveness.

To understand the general behavior of iron release by bacterial activity, it is necessary to consider all together the 25 independent assays run in this study by varying both $\mathrm{NAu}-1 / \mathrm{NAu}-2$ ratio and the particle concentration in order to infer a biodissolution pattern. To this end, the absorbance spectra obtained from each assay were arranged in a 3-way data set (Nontronite concentration $\mathrm{x}$ NAu-1/NAu-2 ratio $\times$ absorbance spectra) and decomposed with the Candecomp/Parafac method (Figure S1). This approach allows singling the spectrum of interest out from the important absorbance background noise resulting from the different components present in the media and their possible interactions. Thus, the decomposition led to the extraction of the mixture coefficients $\mathrm{a}_{f}$ and $\mathrm{b}_{f}$ related to the contribution of the ferrospectral-Fe complex signal to the global absorbance signal at any nontronite concentration and at any [NAu-1]/[NAu-1] + [NAu2] ratio respectively. The iron release patterns from NAu-1 and NAu-2 during the incubation with $P$. aeruginosa PAO1 WT are presented in Figure 2. Overall, the data confirm that (i) iron is released from nontronites in a particle concentration-dependent manner (Figure 2A) and (ii) NAu-2 released substantially greater (approximately 3- to 4-fold) amounts of iron than NAu-1 (Figure 2B).

The same multi-way experiments were run with PAO1 $\triangle p v d A$, a mutant strain unable to synthesize pyoverdine. By contrast to the WT strain, the dissolved Fe was below the detection limit in most of the 25 assays. The fact that no iron was detected after $\mathrm{KCl}$ and hydroxyalamine- $\mathrm{KCl}$ extraction suggested that the absence of detectable $\mathrm{Fe}$ in solution could not be explained by the adsorption or precipitation of Fe on the mineral and/or the silica gel surface. The data imply that the mutant strain was not capable to dissolve significant amounts of Fe(III) from NAu-1 or NAu-2 in our experimental set up (no physical contact between the bacteria cells and the mineral).

\section{Relative Contribution of Organic Acids and Siderophores to the Iron Dissolution Process Organic Acids Profiles}

As shown previously, bacteria can close the concentration gap between mineral solubility and Fe requirement by solubilizing partially iron containing minerals. This process can be achieved 

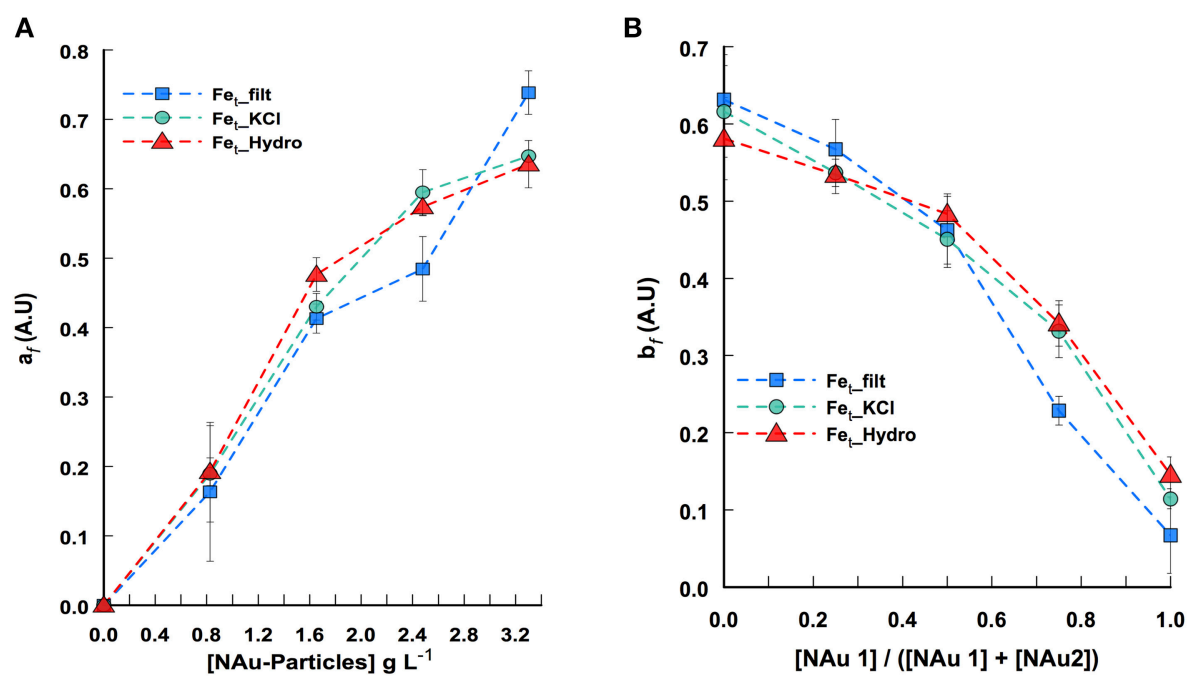

FIGURE 2 | Pattern of iron release determined from Candecomp/Parafac decomposition of the 3-way data set. (A) Fe dissolution as a function of all ratio tested $(1 \geq[\mathrm{NAu}-1] /([\mathrm{NAu}-1]+[\mathrm{NAu}-2] \geq 0)$ across a range of particle concentration. (B) Fe dissolution as a function of all particle concentrations (0-3.3 $\mathrm{g} \mathrm{L}-1)$ tested across a range of NAu-1 and NAu-2 ratios. $\mathrm{a}_{f}$ and $\mathrm{b}_{f}$ indicate the contribution of the ferrospectral-Fe complex signal to the global absorbance at any nontronite concentration and at any NAu-1 to $\mathrm{NAu}-2$ ratio, respectively. The symbols represent the profile of soluble $\mathrm{Fe}$ (squares), $\mathrm{KCl}$ extracted $\mathrm{Fe}$ (circles), hydroxylamine- $\mathrm{KCl}$ extracted Fe (triangles). Error bars indicate the standard error of the mean $(n=2)$.

by lowering the external $\mathrm{pH}$ and/or by the excretion of low molecular weight organic acids able chelate iron (Balland et al., 2010). The analysis of the culture media during the incubation time revealed the presence of oxalate, methanoate, and acetate (Figure 3). Acetate was the main organic molecule released, up to $340 \mathrm{mg} \mathrm{L}^{-1}$, while less than $10 \mathrm{mg} \mathrm{L}^{-1}$ of oxalate was present in the media. The results point out that PAO1 WT and PAO1 $\Delta p v d A$ produced the same low molecular weight organic molecules regardless the crystallographic structure of nontronite (NAu-1 vs. NAu-2). Despite some variations in organic acids profiles (e.g., higher level of acetate released by PAO1 $\Delta p v d A$ compared to the wild-type strain; Figure 3A), the solubilisation of iron cannot be attributed solely to the production of these organic compounds as no Fe was detected in the assays run with PAO1 $\Delta p v d A$. However, we cannot rule out the possibility that they play a role in the dissolution process in the presence of siderophores. Indeed, it has been proposed that oxalate act in conjunction with siderophores and such synergic effect facilitates the dissolution of Fe from sparingly soluble minerals (Kraemer, 2004; Dehner et al., 2010).

\section{Patterns of Pyoverdine Production}

An important route for Fe acquisition in bacteria is the excretion of siderophores. The production of pyoverdine by PAO1 WT was measured in the 25 independent assays. As an example, Figures 4A,B display pyoverdine production profile when the assays were run with a constant nontronite ratio $(100 \%$ of $\mathrm{NAu}-2$ ) across a range of particle concentration and with a constant particle concentration (i.e., $3.3 \mathrm{~g} \mathrm{~L}^{-1}$ ) while varying $\mathrm{NAu}-1$ and NAu-2 ratio, respectively. The highest pyoverdine production level $(20 \pm 1.29 \mu \mathrm{M})$ was observed in the assays run without $\mathrm{Fe}$ (no added mineral) (Figure 4A). On the contrary, a decrease of about $88 \%$ was observed for the higher particle concentration showing that pyoverdine secretion is related to $\mathrm{Fe}$ deficient conditions. The assays run with different NAu-1/NAu2 ratios indicated that the concentration of pyoverdine reached $26.26 \pm 0.91 \mu \mathrm{M}$ in the presence of $100 \%$ of NAu- 1 (Figure 4B). In contrast, this concentration was 8 -fold less when the $\mathrm{Fe}$ source was $100 \%$ of NAu-2. Pyoverdine secretion and Fe release (Figure 1) profiles were similar, thus supporting the critical role played by pyoverdine during the mobilization of structural $\mathrm{Fe}(\mathrm{III})$ as reported previously (Ferret et al., 2014). Additionally, the largest concentration of pyoverdine was measured when $100 \%$ of NAu-1 was used as the sole source of Fe. This result is in agreement with our previous observation (Figure 1) and fully supports our hypothesis that structural Fe(III) is more bioaccessible from NAu-2 than from NAu-1.

The absence of dissolved Fe in the culture medium when the experiments were run with $P$. aeruginosa PAO1 $\Delta p v d A$ and the fact that the same organic acids were produced by the mutant and the wild type cells clearly indicated that pyochelin and organic acid alone were not sufficient to dissolve Fe(III) from nontronite. Although Fe release was observed in the presence of pyoverdine (Figures 1, 4), our results did not allow to conclude whether the observed dissolution was only due to pyoverdine or to the synergic action of pyoverdine, pyochelin and organic acid.

Similar to our approach developed for the analysis of the global profile of Fe release, the absorbance spectra obtained from each assay were arranged in a 3 -way data set (absorbance spectra $\times$ nontronite concentration $\times$ NAu- $1 / \mathrm{NAu}-2$ ratio) from which the pyoverdine secretion pattern can be described after spectral CP decomposition (Figure S1). The decomposition led to the extraction of the mixture coefficients $a_{f}$ and $b_{f}$ related 

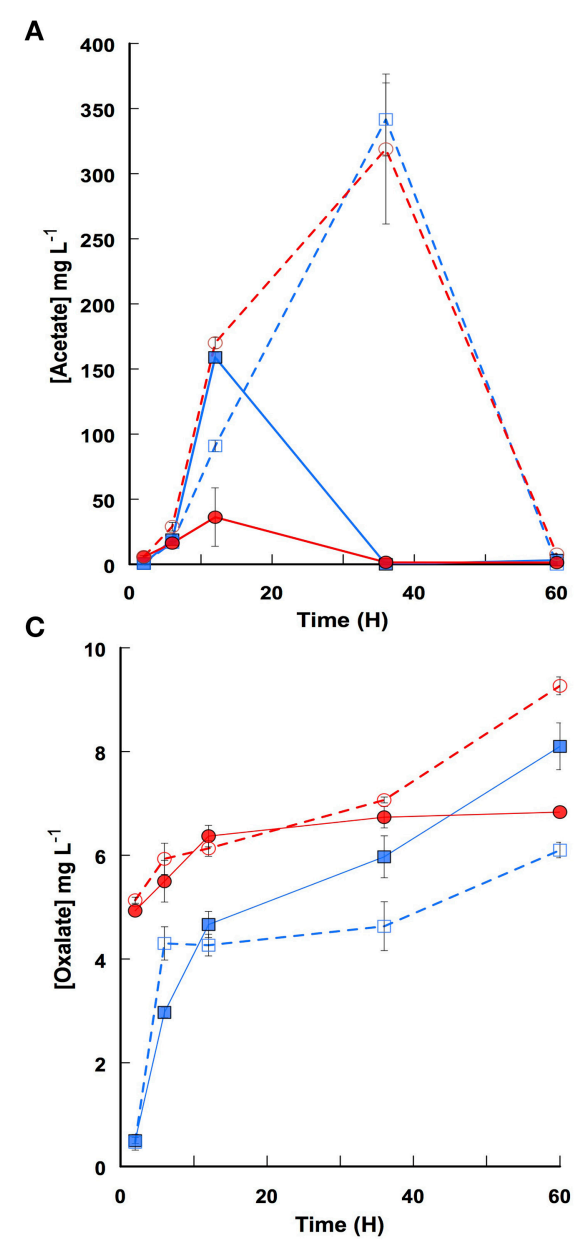

B

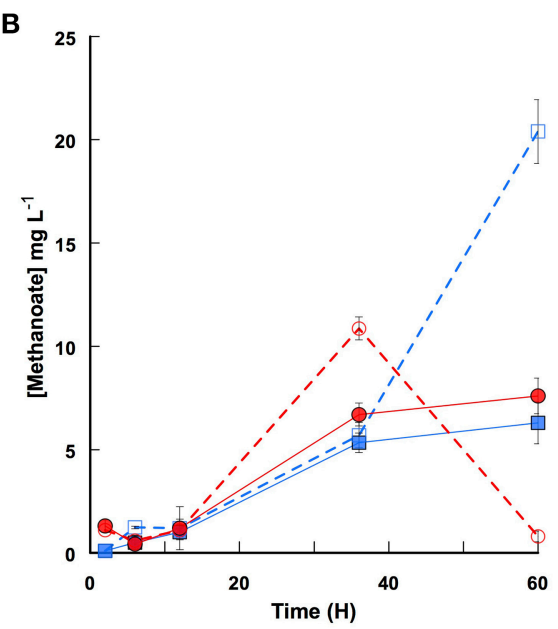

$\square$ PAO1_Wt_NAu 1

- - - - PA01_ $\triangle P v d A \_N A u 1$

O- PAO1_Wt_NAu 2

$--\Theta-\cdot$ PAO1_DpvdA_NAu 2

FIGURE 3 | Time profiles of acetate (A), methanoate (B), and oxalate (C) production by PAO1 WT (filled symbols) and PAO1 $\triangle$ pvdA (empty symbols) in a medium containing NAu-1 (squares) and NAu-2 (circles). Values are means of triplicate measurements.

to the contribution of the pyoverdine signal to the global absorbance signal at any nontronite concentration and at any $[\mathrm{NAu}-1] /[\mathrm{NAu}-1]+[\mathrm{NAu} 2]$ ratio, respectively (Figures 4C,D). The pyoverdine production profile $\mathrm{a}_{f}$ as the function of particle concentration at any $\mathrm{NAu}-1 / \mathrm{NAu}-2$ ratio decreased with particle concentration (Figure 4C). The pattern of pyoverdine secretion $\mathrm{b}_{f}$ for the different $\mathrm{NAu}-1 / \mathrm{NAu}-2$ ratio tested at any particle concentration indicated a slight increase in the range of 100 to $50 \%$ of NAu- 2 and a sharp rise, with the highest production in the presence of $100 \%$ of NAu-1 (Figure 4D), which is in well line with the iron dissolution profile determined above (Figure 2B). Even qualitative, the CP analysis provides a means of displaying the general profile of pyoverdine secretion in which the combined effect of both particle concentration and NAu-1/NAu-2 ratio variation were taken into account. Overall these results indicate that the mixing of NAu-1 and NAu2 at different ratios did not affect the pyoverdine production profile due to a combined effect between the two minerals that could have been different from the sum of the individual effect.

\section{Abiotic Dissolution of Iron}

To further investigate the role of pyoverdine in the mobilization of iron from nontronite, we performed batch experiments where silica gel plugs containing NAu-2 were incubated in iron deficient DCAA medium supplemented with purified pyoverdine, as well as in cell-free culture supernatants of PAO1 WT grown in DCAA. The analysis of the assay media after $24 \mathrm{~h}$ of incubation revealed that iron was mobilized in both cases (Figure 5). Comparable initial concentrations of pyoverdine in the assays led to the same level of iron dissolution, indicating that organic acids, pyochelin or other metabolite in the culture supernatant did not significantly contribute to mineral weathering. This was confirmed by the absence of detectable iron in assays performed with culture supernatants of the pyoverdin deficient mutant PAO1 $\Delta p v d D$ and the double pyochelin- and pyoverdine-deficient mutant (PAO1 $\triangle p v d D \triangle p c h E F$ ). Overall, these data unambiguously show that pyoverdine is the main agent involved in weathering of nontronite in the absence of physical contact with bacterial cells. 

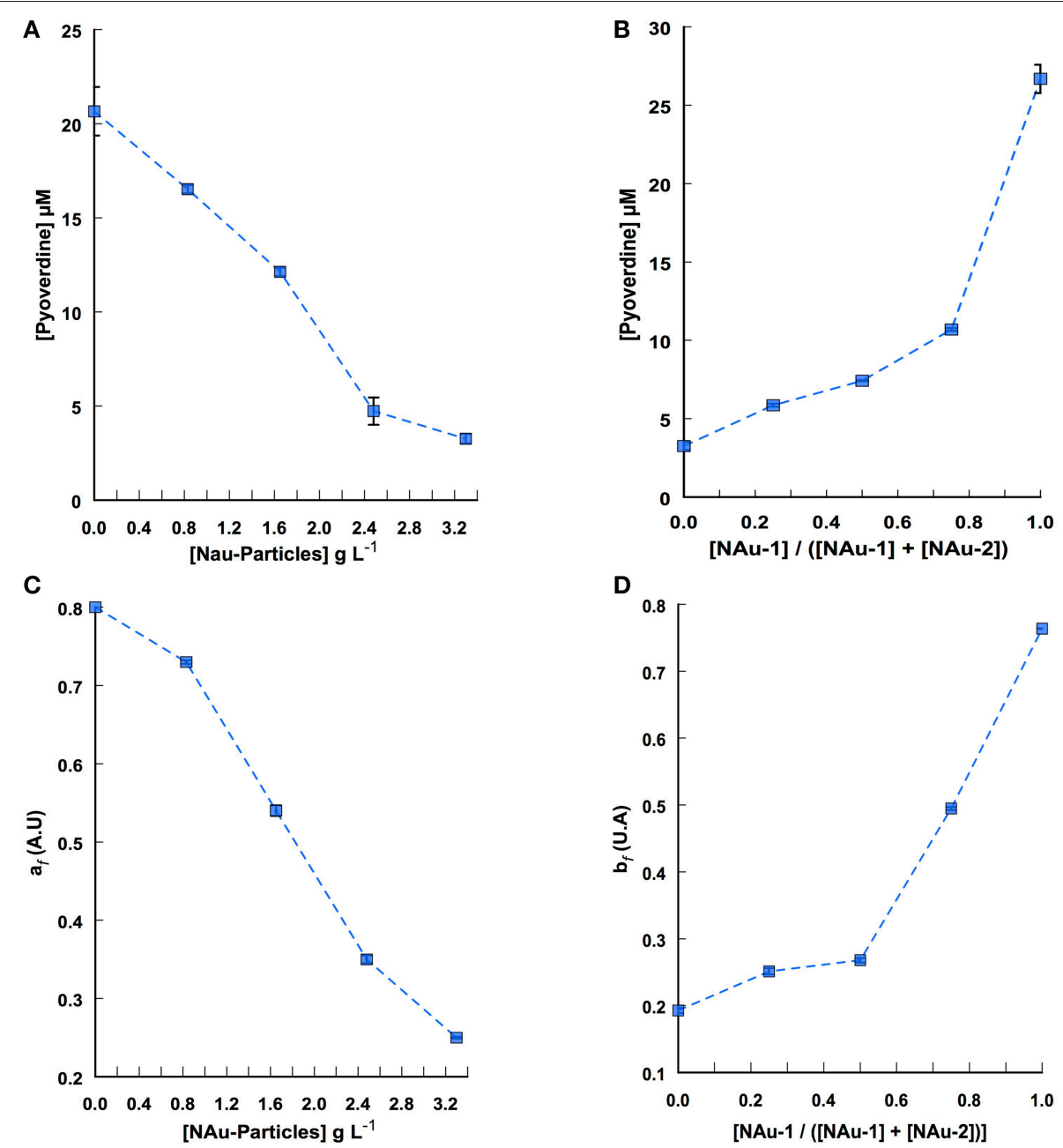

FIGURE 4 | Pyoverdine production by $P$. aeruginosa PAO1 incubated for $24 \mathrm{~h}$ in DCAA medium with increasing nontronite particle concentration and variables NAu-1 to NAu-2 ratios. (A) Pyoverdine production in assays where the NAu-1 and NAu-2 ratio is kept constant ([NAu-1]/([NAu-1] + [NAu-2]) $=0)$ while the particle concentration is varied. (B) Pyoverdine production in assays where the particle concentration is kept constant $\left(3.3 \mathrm{~g} \mathrm{~L}^{-1}\right)$ with different NAu-1 to NAu-2 ratios. (C) Pattern of pyoverdine production as a function of all tested ratios over a range of particle concentrations, determined from CP decomposition of the 3-way data set. (D) Pattern of pyoverdine production as a function of all particle concentrations tested over a range of NAu- 1 and NAu- 2 ratios, determined from CP decomposition. $a_{f}$ and $b_{f}$ indicate the contribution of the pyoverdine signal to the global absorbance signal at any nontronite concentration and at any NAu- 1 to NAu-2 ratio, respectively. Error bars represent the standard error of the mean $(n=2)$, most of them are within the size of the symbols.

A unique advantage of our bioweathering assay is that the size-defined, monolayer nontronite particles are homogeneously dispersed in the silica gel matrix, meaning they are evenly accessible to bacterial weathering metabolites. This feature makes it possible to infer a possible dissolution scenario from the knowledge of nontronite structure and above experimental data. Actually, in weathering assays run with NAu-2, only about $0.1 \%$ of the total structural iron was released in the medium, which corresponds to iron accessible from mineral edge sites. Previous acid dissolution experiments (e.g., Bickmore et al., 2001; Grybos et al., 2010) showed that the edge surfaces of phyllosilicates, characterized by groups with broken bonds, are much more reactive than the basal planes, which are built up with chargesatisfied and stable siloxane bonds. Edge surface sites also appear to be preferentially attacked by organic ligands (Bray et al., 2015; Zhang and Jun, 2015). This suggests that siderophore-promoted nontronite dissolution is dominated by cristal edges. In addition, the more extensive iron release from NAu-2 also is most likely due to a higher occurrence of tetrahedral $\mathrm{Fe}(\mathrm{III})$ in the structure compared to NAu-1 (8 and $2 \%$ respectively). We presume either that $\mathrm{Fe}$ (III) in tetrahedra is more accessible to chelating siderophores than in octahedra, or that dissolution of tetrahedral $\mathrm{Fe}(\mathrm{III})$ promotes iron release form adjacent octahedron sites via a yet unidentified mechanism.

Here we chose to work with a given particle size to assess the biological weathering processes independently of the possible contribution of basal to edge surface ratio, which depends on particle size (Grybos et al., 2011). The impact of different particle 


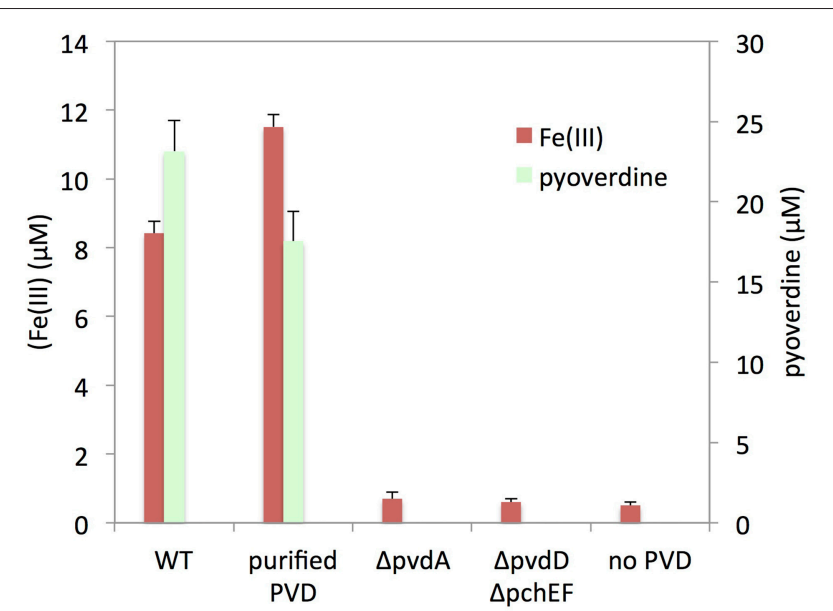

FIGURE 5 | Pyoverdine-mediated iron release from NAu-2. Silica gel embedded NAu-2 particles were incubated with or without purified pyoverdine (PVD) and with cell-free culture supernatants of PAO1 WT, PAO1 $\triangle p v d A$ or PAO1 $\Delta p v d D \triangle p c h E F$ strains grown in DCAA medium. Pyoverdine and iron concentrations were determined after $24 \mathrm{~h}$ of incubation. Errors bars represent the standard error of the mean $(n=3)$.

sizes on the extent of iron dissolution is now being investigated to define whether siderophores have a preferential reactive site.

\section{Effect of Physical Contact on Fe Acquisition}

To test whether a direct contact would alleviate the need for pyoverdine, a $b f r B-g f p$ gene fusion constructed in pPROBE-NT was introduced into $P$. aeruginosa PAO1 $\triangle p v d D \triangle p c h E F$, The expression of the $b f r B$ gene encoding a bacterioferritin involved in iron storage is induced under iron-replete conditions and, therefore, high expression levels of this gene would indicate that the cells satisfied their metabolic Fe requirement (Parrello et al., 2015). P. aeruginosa $\triangle p v d D \triangle p c h E F /$ pPROBE-bfrB was incubated in direct contact with NAu-1 and NAu-2. The induction of $b f r B$ gene expression was estimated by measuring the GFP fluorescence after $24 \mathrm{~h}$ of incubation (Figure 6). The images of bacterial cell aggregates show an expression of $b f r B$ gene in the presence of nontronite, with a higher expression level when the experiment was run with NAu-2 (Figure 6B) compared to NAu-1. This reinforced our previous observations (Figures 1, 4) that the bioavailability of Fe is controlled by the crystallography of the mineral. Furthermore, the siderophoredeficient mutant was able to acquire $\mathrm{Fe}$, which was not readily available for biological uptake, when a physical contact was established with the mineral. A simplified scheme for Fe release from iron bearing clays such as nontronites is proposed in Figure 7.

Previous work with a siderophore-deficient mutant of Pseudomonas mendocina ymp resulted in similar observations. The mutant strain was unable to acquire $\mathrm{Fe}$ from iron containing minerals without a physical contact (Dehner et al., 2011; Kuhn et al., 2013). However, the incubation of cells

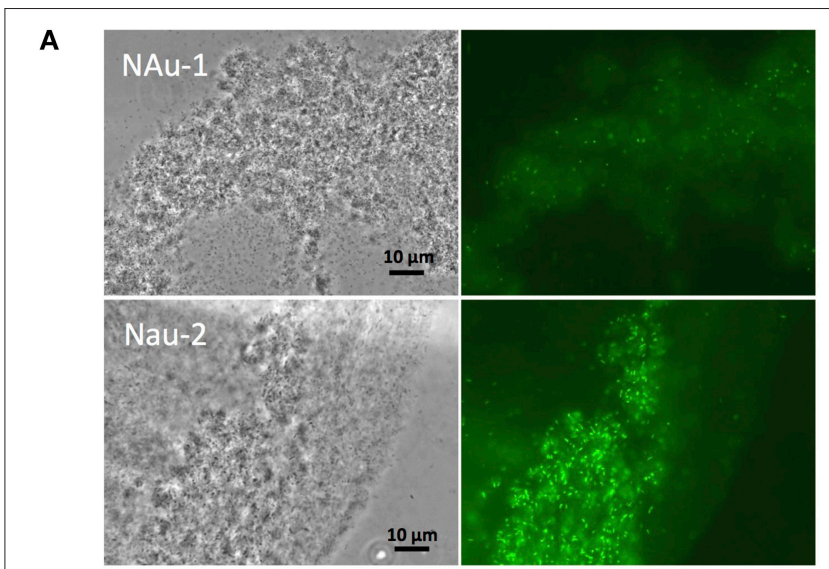

B

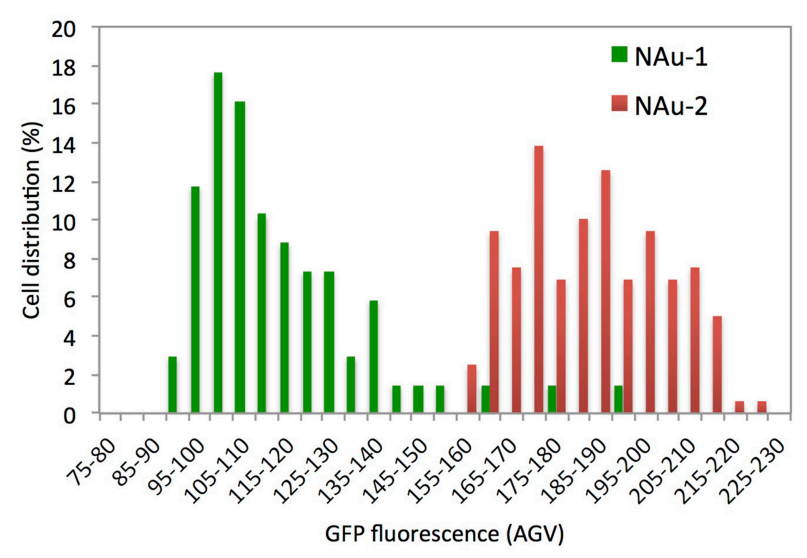

FIGURE 6 | Fluorescence of siderophore deficient $\boldsymbol{P}$. aeruginosa strain PAO1 $\triangle p v d D \Delta p c h E F$ containing an iron regulated bfrB-gfp gene fusion incubated in direct contact with nontronite particles. (A) Representative transmission (left panels) and fluorescence (right panels) micrographs showing aggregation of bacteria with NAu-1 (top panels) and NAu-2 (bottom panels) particles. (B) Expression of GFP in individual cells determined from analysis of images captured by fluorescence microscopy.

in the vicinity of the mineral led to the acquisition of $\mathrm{Fe}$ from the mineral phase, indicating that another metabolic strategy for iron uptake could be activated to alleviate the absence of siderophore. Indeed, Dehner and co-workers have demonstrated that Pseudomonas mendocina was able to reduce and withdraw $\mathrm{Fe}$ from montmorillonite, an iron containing clay mineral via a cell-associated enzyme. Similarly, $P$. aeruginosa could use a reduction-promoted Fe acquisition when the cells were in direct contact with Fe bearing minerals.

\section{CONCLUSION}

$P$. aeruginosa is capable to acquire structural Fe(III) of a clay mineral, nontronite, through the use of siderophores when there is a physical barrier between the cells and the mineral particles. Furthermore, the mixing of NAu-1 and NAu-2 didn't affect neither the release of $\mathrm{Fe}(\mathrm{III})$ nor the production of 


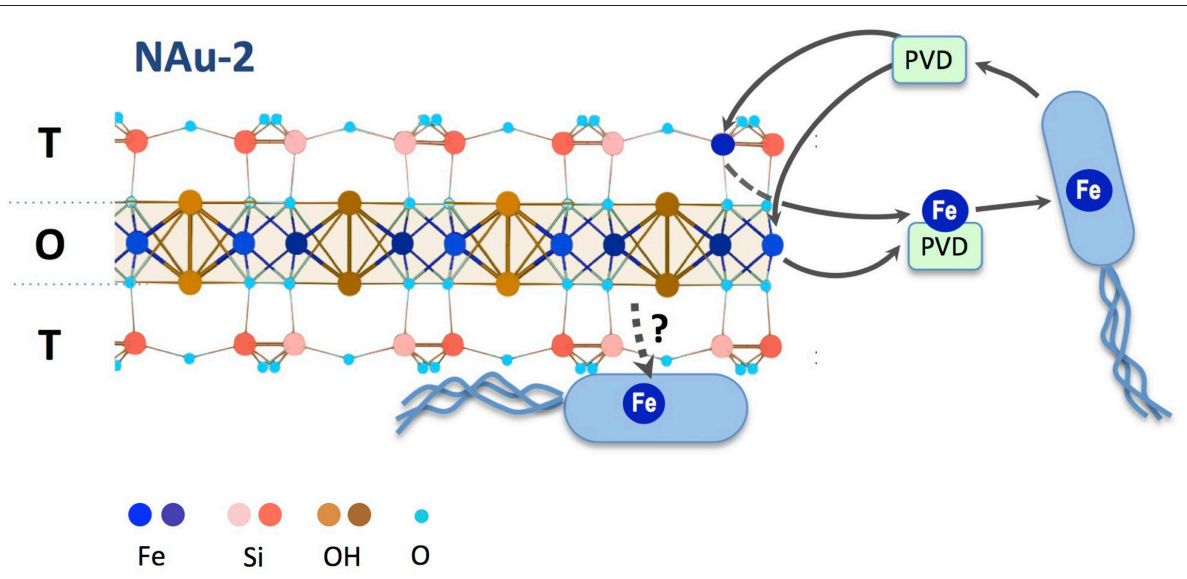

FIGURE 7 | Proposed mechanism of iron mobilization from nontronite. Under iron deficient conditions, $P$. aeruginosa produces pyoverdine that chelates and solubilizes Fe(III) from edge sites of octahedral (O) or tetrahedral (T) sheets. The higher occurrence of tetrahedral Fe(III) sites in NAu-2 facilitates iron mobilization compared to NAu-1. Iron from pyoverdine- $\mathrm{Fe}^{3+}$ complexes can be taken up into the bacterial cell to sustain growth. When in direct contact with the mineral particles, $P$. aeruginosa can acquire iron via a siderophore-independent mechanism.

siderophore indicating the absence of interaction between NAu1 and NAu-2 as shown by our multi-parametric experimental approach coupled to CP spectral decomposition. Although $P$. aeruginosa can produce two siderophores, pyoverdine and pyochelin, pyoverdine is the key driver of Fe solubilisation. A $P$. aeruginosa mutant deficient in the production of both siderophores appears to acquire $\mathrm{Fe}$ from nontronite if a physical contact between the bacterial cells and the mineral is established. This second mechanism of Fe acquisition is still under investigation. Structural Fe(III) of NAu-2 was shown to be more bioaccessible than the one of NAu-1 independently of the Fe acquisition pathway (i.e., pyoverdine or direct contact-promoted Fe acquisition). Thus, the bioavailability of structural $\mathrm{Fe}$ (III) is governed by the crystallochemistry of the mineral. This observation leads to questions how the crystal chemical environment of $\mathrm{Fe}$ (III) (tetrahedron, octahedron) constrains the release of $\mathrm{Fe}$ and how siderophores interact with these sites. Furthermore, it is established that bacteria can influence layer charge, cation exchange capacity, swelling and the rheological properties of clay minerals through the production of siderophores. Therefore, additional work is needed with different particle sizes and different environmental clay samples to constrain how the change in their properties and behavior

\section{REFERENCES}

Albrecht-Gary, A. M., and Crumbliss, A. L. (1998). Coordination chemistry of siderophores: thermodynamics and kinetics of iron chelation and release. Met. Ions Biol. Syst. 35, 239-327.

Albrecht-Gary, A.-M., Blanc, S., Rochel, N., Ocaktan, A. Z., and Abdallah, M. A. (1994). Bacterial iron transport: coordination properties of pyoverdin PaA, a peptidic siderophore of pseudomonas aeruginosa. Inorg. Chem. 33, 6391-6402. doi: 10.1021/ic00104a059

Andrews, S. C., Robinson, A. K., and Rodríguez-Quiñones, F. (2003). Bacterial iron homeostasis. FEMS Microbiol. Rev. 27, 215-237. doi: 10.1016/S01686445(03)00055-X induced by bacterial activity impact the biogeochemical cycle of iron and associated trace elements.

\section{AUTHOR CONTRIBUTIONS}

Conceived and designed experiments: DP, CM, PB, AZ. Performed the experiments: DP, CM, PB, AZ. Analyzed the data: DP, CM, PB, AZ. Wrote the paper: AZ, PB. All authors read and approved the final manuscript.

\section{ACKNOWLEDGMENTS}

This work was supported by the French Agence Nationale de la Recherche (ANR) project HÆSPRI (ANR-09-BLAN-0336-04). DP was a recipient of a grant from the same program. We thank Géraldine Kitzinger for her assistance during organic acid analysis.

\section{SUPPLEMENTARY MATERIAL}

The Supplementary Material for this article can be found online at: http://journal.frontiersin.org/article/10.3389/fmicb. 2016.00423

Balland, C., Poszwa, A., Leyval, C., and Mustin, C. (2010). Dissolution rates of phyllosilicates as a function of bacterial metabolic diversity. Geochim. Cosmochim. Acta 74, 5478-5493. doi: 10.1016/j.gca.2010. 06.022

Banwart, S., Davies, S., and Stumm, W. (1989). The role of oxalate in accelerating the reductive dissolution of hematite $(\alpha-\mathrm{Fe} 2 \mathrm{O} 3)$ by ascorbate. Colloids Surf. 39, 303-309. doi: 10.1016/0166-6622(89)80281-1

Bickmore, B. R., Bosbach, D., Hochella, M. F., Charlet, L., and Rufe, E. (2001). In situ atomic force microscopy study of hectorite and nontronite dissolution: implications for phyllosilicate edge surface structures and dissolution mechanisms. Am. Mineral. 86, 411-423. doi: 10.2138/am-20 01-0404 
Brandel, J., Humbert, N., Elhabiri, M., Schalk, I. J., Mislin, G. L. A., and AlbrechtGary, A.-M. (2012). Pyochelin, a siderophore of Pseudomonas aeruginosa: physicochemical characterization of the iron(III), copper(II) and zinc(II) complexes. Dalton Trans. Camb. Engl. 41, 2820-2834. doi: 10.1039/c1dt11804h

Bray, A. W., Oelkers, E. H., Bonneville, S., Wolff-Boenisch, D., Potts, N. J., Fones, G., et al. (2015). The effect of $\mathrm{pH}$, grain size, and organic ligands on biotite weathering rates. Geochim. Cosmochim. Acta 164, 127-145. doi: 10.1016/j.gca.2015.04.048

Cheah, S.-F., Kraemer, S. M., Cervini-Silva, J., and Sposito, G. (2003). Steady-state dissolution kinetics of goethite in the presence of desferrioxamine $B$ and oxalate ligands: implications for the microbial acquisition of iron. Chem. Geol. 198, 63-75. doi: 10.1016/\$0009-2541(02)00421-7

Cornell, R. M., and Schwertmann, U. (2004). "Solubility," in The Iron Oxides (Wiley-VCH Verlag GmbH \& Co. KGaA), 201-220. Available online at: http:// dx.doi.org/10.1002/3527602097.ch9

Cox, C. D., Rinehart, K. L. Jr., Moore, M. L., and Cook, J. C. Jr. (1981). Pyochelin: novel structure of an iron-chelating growth promoter for Pseudomonas aeruginosa. Proc. Natl. Acad. Sci. U.S.A. 78, 4256-4260.

Dehner, C. A., Awaya, J. D., Maurice, P. A., and DuBois, J. L. (2010). Roles of siderophores, oxalate, and ascorbate in mobilization of iron from hematite by the aerobic bacterium Pseudomonas mendocina. Appl. Environ. Microbiol. 76, 2041-2048. doi: 10.1128/AEM.02349-09

Dehner, C. A., Barton, L., Maurice, P. A., and DuBois, J. L. (2011). Size-dependent bioavailability of hematite (alpha- $\mathrm{Fe}_{2} \mathrm{O}_{3}$ ) nanoparticles to a common aerobic bacterium. Environ. Sci. Technol. 45, 977-983. doi: 10.1021/es102922j

Ferret, C., Sterckeman, T., Cornu, J.-Y., Gangloff, S., Schalk, I. J., and Geoffroy, V. A. (2014). Siderophore-promoted dissolution of smectite by fluorescent Pseudomonas. Environ. Microbiol. Rep. 6, 459-467. doi: 10.1111/17582229.12146

Ghysels, B., Dieu, B. T. M., Beatson, S. A., Pirnay, J.-P., Ochsner, U. A., Vasil, M. L., et al. (2004). FpvB, an alternative type I ferripyoverdine receptor of Pseudomonas aeruginosa. Microbiol. Read. Engl. 150, 1671-1680. doi: 10.1099/mic.0.27035-0

Grybos, M., Billard, P., Desobry-Banon, S., Michot, L. J., Lenain, J.-F., and Mustin, C. (2011). Bio-dissolution of colloidal-size clay minerals entrapped in microporous silica gels. J. Colloid Interface Sci. 362, 317-324. doi: 10.1016/j.jcis.2011.07.031

Grybos, M., Michot, L. J., Skiba, M., Billard, P., and Mustin, C. (2010). Dissolution of anisotropic colloidal mineral particles: evidence for basal surface reactivity of nontronite. J. Colloid Interface Sci. 343, 433-438. doi: 10.1016/j.jcis.2009.11.058

Haack, E. A., Johnston, C. T., and Maurice, P. A. (2008). Mechanisms of siderophore sorption to smectite and siderophore-enhanced release of structural Fe3+. Geochim. Cosmochim. Acta 72, 3381-3397. doi: 10.1016/j.gca.2008.03.027

Hider, R. C., and Kong, X. (2010). Chemistry and biology of siderophores. Nat. Prod. Rep. 27, 637-657. doi: 10.1039/B906679A

Kraemer, S. (2004). Iron oxide dissolution and solubility in the presence of siderophores. Aquat. Sci. 66, 3-18. doi: 10.1007/s00027-003-0690-5

Kuhn, K. M., DuBois, J. L., and Maurice, P. A. (2013). Strategies of aerobic microbial Fe acquisition from Fe-bearing montmorillonite clay. Geochim. Cosmochim. Acta 117, 191-202. doi: 10.1016/j.gca.2013.04.028
Meyer, J. M., and Abdallah, M. A. (1978). The fluorescent pigment of pseudomonas fluorescens: biosynthesis, purification and physicochemical properties. Microbiology 107, 319-328.

Michot, L. J., Bihannic, I., Maddi, S., Baravian, C., Levitz, P., and Davidson, P. (2008). Sol/gel and isotropic/nematic transitions in aqueous suspensions of natural nontronite clay. Influence of particle anisotropy. 1. Features of the $\mathrm{i} / \mathrm{n}$ transition. Langmuir ACS J. Surf. Colloids 24, 3127-3139. doi: 10.1021/la703506z

Miller, W. G., Leveau, J. H. J., and Lindow, S. E. (2000). Improved gfp and inaZ broad-host-range promoter-probe vectors. Mol. Plant. Microbe Interact. 13, 1243-1250. doi: 10.1094/MPMI.2000.13.11.1243

Ochsner, U. A., Wilderman, P. J., Vasil, A. I., and Vasil, M. L. (2002). GeneChip expression analysis of the iron starvation response in Pseudomonas aeruginosa: identification of novel pyoverdine biosynthesis genes. Mol. Microbiol. 45, 1277-1287. doi: 10.1046/j.1365-2958.2002.03084.x

Oulkadi, D., Balland-Bolou-Bi, C., Billard, P., Kitzinger, G., Parrello, D., Mustin, C., et al. (2014). Interactions of three soil bacteria species with phyllosilicate surfaces in hybrid silica gels. FEMS Microbiol. Lett. 354, 37-45. doi: 10.1111/1574-6968.12421

Parrello, D., Mustin, C., Brie, D., Miron, S., and Billard, P. (2015). Multicolor whole-cell bacterial sensing using a synchronous fluorescence spectroscopybased approach. PLoS ONE 10:e122848. doi: 10.1371/journal.pone.01 22848

Powell, P. E., Cline, G. R., Reid, C. P. P., and Szaniszlo, P. J. (1980). Occurrence of hydroxamate siderophore iron chelators in soils. Nature 287, 833-834. doi: 10.1038/287833a0

Saha, R., Saha, N., Donofrio, R. S., and Bestervelt, L. L. (2013). Microbial siderophores: a mini review. J. Basic Microbiol. 53, 303-317. doi: 10.1002/jobm.201100552

Schalk, I. J., and Guillon, L. (2013). Pyoverdine biosynthesis and secretion in Pseudomonas aeruginosa: implications for metal homeostasis. Environ. Microbiol. 15, 1661-1673. doi: 10.1111/1462-2920.12013

Visca, P., Colotti, G., Serino, L., Verzili, D., Orsi, N., and Chiancone, E. (1992). Metal regulation of siderophore synthesis in Pseudomonas aeruginosa and functional effects of siderophore-metal complexes. Appl. Environ. Microbiol. 58, 2886-2893.

Zhang, L., and Jun, Y.-S. (2015). Distinctive reactivities at biotite edge and basal planes in the presence of organic ligands: implications for organicrich geologic $\mathrm{CO}_{2}$ sequestration. Environ. Sci. Technol. 49, 10217-10225. doi: 10.1021/acs.est.5b01960

Conflict of Interest Statement: The authors declare that the research was conducted in the absence of any commercial or financial relationships that could be construed as a potential conflict of interest.

Copyright $\odot 2016$ Parrello, Zegeye, Mustin and Billard. This is an open-access article distributed under the terms of the Creative Commons Attribution License (CC BY). The use, distribution or reproduction in other forums is permitted, provided the original author(s) or licensor are credited and that the original publication in this journal is cited, in accordance with accepted academic practice. No use, distribution or reproduction is permitted which does not comply with these terms. 